\title{
TRAIT ANXIETY, DEFENSIVENESS, AND THE STRUCTURE OF WORRY
}

\author{
Michael W. EysenCK ${ }^{1 *}$ and Jos VAN BerKum ${ }^{2}$ \\ ${ }^{1}$ Department of Psychology, Royal Holloway and Bedford New College, University of London, Egham, \\ Surrey TW20 OEX, England and ${ }^{2}$ Institute of Experimental Psychology, University of Groningen, \\ The Netherlands
}

(Received 31 January 1992)

\begin{abstract}
Summary-A principal components analysis of the ten scales of the Worry Questionnaire revealed the existence of major worry factors or domains of social evaluation and physical threat, and these factors were confirmed in a subsequent item analysis. Those high in trait anxiety had much higher scores on the Worry Questionnaire than those low in trait anxiety, especially on those scales relating to social evaluation. Scores on the Marlowe-Crowne Social Desirability Scale were negatively related to worry frequency. However, groups of low-anxious and repressed individuals formed on the basis of their trait anxiety and social desirability scores did not differ in worry. It was concluded that worry, especially in the social evaluation domain, is of fundamental importance to trait anxiety.
\end{abstract}

There has been increased interest in recent years in the phenomenon of worry (see Eysenck, 1992, for review). Worry has been defined in various ways. For example, Borkovec, Robinson, Pruzinsky and DePree (1983) proposed the following definition: "Worry is a chain of thoughts and images, negatively affect-laden and relatively uncontrollable" (p. 9). In more general terms, some theorists (e.g. Eysenck, 1992; Steptoe \& Kearnsley, 1990) have argued that worry is basically the cognitive component of anxiety, and that is the position which will be adopted here.

There are several reasons for claiming that there is a close relationship between worry and anxiety. Of particular relevance to the study reported here is evidence indicating that individuals high in the personality dimension of trait anxiety worry considerably more than those low in trait anxiety. For example, Borkovec et al. (1983) obtained a correlation of +0.67 between trait anxiety and the amount of time which people spend worrying. Tallis, Eysenck and Mathews (1992) reported a correlation of +0.76 between trait anxiety and a worry questionnaire, and of +0.84 between trait anxiety and a slightly modified version of the same questionnaire.

More evidence relating anxiety and worry comes from the third revised Diagnostic and Statistical Manual (DSM-III-R) of the American Psychiatric Association. According to DSM-III-R, the presence of prolonged (more than 6 months), unrealistic and excessive worry is found in generalized anxiety disorder. Such worry is the central defining feature of generalized anxiety disorder.

Various attempts have been made to ascertain the major domains of worry. Meyer, Miller, Metzger and Borkovec (1990) devised the Penn State Worry Questionnaire. This questionnaire assesses only a general worry factor, in part because previous versions of the questionnaire had failed to reveal the existence of replicable specific worry factors. However, this failure to identify specific worry domains appears to depend at least in part on the kinds of questions included in the questionnaire. For example, many of the items included in the Penn State Worry Questionnaire are very general in nature (e.g. 'I have been a worrier all my life'). With such items, it is unsurprising that only a general factor could be identified.

Barlow (1988) identified four worry spheres or domains on the basis of the unrealistic worries reported by generalized anxiety disorder patients: family; finance; work; and personal illness. Some evidence that these spheres are also applicable to normal groups was obtained by Craske, Rapee, Jackel and Barlow (1989). However, while Barlow's (1988) approach is reasonable, it is rather limited in that it focuses on the external sources of worry at the expense of the internal psychological factors.

*To whom correspondence should be addressed. 
Tallis et al. (1992) analyzed the data from a worry questionnaire by means of an agglomerative method of cluster analysis, and reported six clusters or worry domains: relationships; lack of confidence; aimless future; work incompetence; financial concerns; and socio-political concerns. However, there were reasonably high inter-correlations among most of these worry domains, suggesting that the actual number of separate worry domains may be less than six.

This study was designed in part to investigate the issue of whether there is a single general worry factor or whether there are several worry domains. It was also designed to establish more fully the relationship between trait anxiety and worry. It has been suggested (e.g. Weinberger, Schwartz \& Davidson, 1979) that individuals scoring low on trait anxiely form a rather helerogeneous group. Those who score low on the Marlowe-Crowne Social Desirability Scale (Crowne \& Marlowe, 1964) as well as on measures of trait anxiety have been termed low-anxious, whereas those who score high on the Marlowe-Crowne but low on trait anxiety have been termed repressors. Even though low-anxious $S$ s and repressors have comparably low scores on trait anxiety, Weinberger et al. (1979) discovered on a somewhat stressful task that repressors were more stressed than low-anxious individuals on six different measures (three of which were physiological and the remainder of which were based on task performance). In similar fashion, Schwartz (1983) measured alpha-EEG activity under resting conditions, and discovered that repressors were more aroused than those high in trait anxiety, whereas low-anxious $S$ s were much less aroused than either of the other two groups.

In view of the above evidence, it was decided to see whether repressors and low-anxious individuals differed in terms of worry. Since repressors are in a sense more stressed than low-anxious individuals, it might be expected that this would be reflected in higher reported worry scores. On the other hand, repressors typically deny the existence of negative thoughts and stress, and so it seemed more likely that repressors would report very low levels of worrying, possibly even lower than those of genuinely low-anxious individuals.

\section{METHOD}

\section{Subjects}

The $S$ s were 113 adults recruited from a part-time college, from a register of participants in psychological experiments, from an insurance company, and from two publishing firms. They had a mean age of 32.3 years ( $S D=9.0$ years). In order to explore fully the relationship between personality and worry, three subgroups were formed for some of the analyses. High defensiveness was defined as scores in the upper quartile of scores (above 16) on the Marlowe-Crowne Social Desirability Scale (Crowne \& Marlowe, 1964) and low defensiveness was defined as lying within the lower half of scores on that scale (13 or less). A comparable upper quartile vs lower half categorization was applied to scores on the trait anxiety scale of Spielberger's State-Trait Anxiety Inventory (Spielberger, 1983), producing cut-offs of 50 and 13, respectively. Those with high defensiveness scores and low trait anxiety scores were assigned to the repressor group $(n=21)$; those with low defensiveness scores and high trait anxiety scores were allocated to the high-anxious group $(n=21)$; and those with low defensiveness scores and low trait anxiety scores were assigned to the low-anxious group $(n=23)$. Very few $S$ s fell in the high defensiveness, high trait anxiety group.

\section{Worry Questionnaire}

A 55-item Worry Questionnaire was constructed on the basis of previous work by Mathews and MacLeod (see Eysenck \& Mathews, 1987). A total of 109 worry items were cluster-analyzed using the inter-item correlations as the similarity measure, and the group average linkage procedure as the hierarchical clustering method. This produced nine clusters which appeared to have psychological reality as worry domains: health; general social evaluation; personal relationships; finance; personal fulfilment; nuclear-international concerns; societal and environmental concerns; physical health of close ones; and physical appearance. Each of these domains was represented by five items. Two more sets of five items were added on the basis of additional unpublished work by Mathews and MacLeod: one set related to physical health concerns beyond one's control, and the other set to panic and loss of control. In fact, the health and physical health concerns beyond one's control formed overlapping domains, and were combined to form a physical health domain. Thus, the 
Worry Questionnaire assessed ten worry domains. There was a worry frequency scale (How frequently does this concern, or one very similar to it, tend to pass through your mind?), and a worry intensity scale (How upsetting is a typical occurrence of this worry?).

\section{Procedure}

$S$ s were presented with a short overview of the experiment. They were then required to complete the Worry Questionnaire, the trait anxiety scale of the State-Trait Anxiety Inventory, and the Marlowe-Crowne Social Desirability Scale. Finally, they provided answers to questions relating to age, sex, and occupation.

\section{RESULTS}

The mean worry frequency across all items and all $S$ s was $1.51(\mathrm{SD}=0.84)$. In order to interpret this mean, it should be noted that an item which was never worried about received a rating of 0 , and one which was worried about once a month received a rating of 2 . Mean worry frequency correlated significantly with trait anxiety, $r=+0.65, P<0.001$, and it also correlated significantly with defensiveness as assessed by the Marlowe-Crowne Social Desirability Scale, $r=-0.26$, $P<0.005$. However, mean worry frequency did not correlate with age, $r=+0.01$. The two personality measures (i.e. trait anxiety and defensiveness) correlated significantly with each other, $r=-0.37, P<0.001$. Inter-correlations involving worry intensity are not given for reasons discussed below.

\section{Worry Questionnaire}

The correlation between mean worry frequency and mean worry intensity was $+0.73, P<0.001$. The correlations between frequency and intensity for each individual item were generally substantial, with only 8 out of 55 items having correlations falling below $r=+0.60$. The mean item frequency-intensity correlation was +0.69 . There was a minor problem with the intensity data, in that some $S$ s gave a non-zero intensity rating to items which they claimed never to worry about. As a consequence, all of the subsequent analyses of the Worry Questionnaire were based on the frequency rather than the intensity data.

The worry frequency data for each of the ten scales or domains of the Worry Questionnaire were inter-correlated. As can be seen in Table 1, several of the scales were highly inter-correlated. As a consequence, data from the ten scales were submitted to a principal components analysis, with the two resulting factors being varimax-rotated. The first factor had an eigenvalue of 5.23 , and accounted for $52.3 \%$ of the variance. As can be seen in Table 2, the main loadings on this factor were from the general social evaluation, personal fulfilment, personal relationships, and finance scales; these loadings suggest that the factor is primarily concerned with social evaluation. The second factor had an eigenvalue of 1.14 , and accounted for $11.4 \%$ of the variance. As can be seen in Table 2, the main loadings on this factor were from the physical health of close ones, social and environmental concerns, nuclear-international concerns, and physical health scales; these loadings suggest that the factor is mainly concerned with physical threat.

A further principal components analysis was carried out on all 55 items in the Worry Questionnaire. This analysis yielded five factors with an eigenvalue of $>2$. These factors were then varimax-rotated, with the result that the first three factors were interpretable. The first factor had an eigenvalue of 14.85 , and accounted for $27.0 \%$ of the variance. It was essentially a social evaluative factor. The second factor had an eigenvalue of 4.04 , and accounted for $7.3 \%$ of the variance. It was a physical threat factor. The third factor had an eigenvalue of 2.27 , and accounted for $5.2 \%$ of the variance. All of the 8 items which loaded more than 0.50 on this factor were from the domains of nuclear and international concerns or societal and environmental concerns, thus defining the factor as one of societal problems.

\section{Personality}

A two-way analysis of variance was conducted on the overall worry frequency data from the Worry Questionnaire; the two factors were personality group and gender. There was a non-significant main effect of gender, $F(1,59)=0.08$, and the interaction between gender and personality 

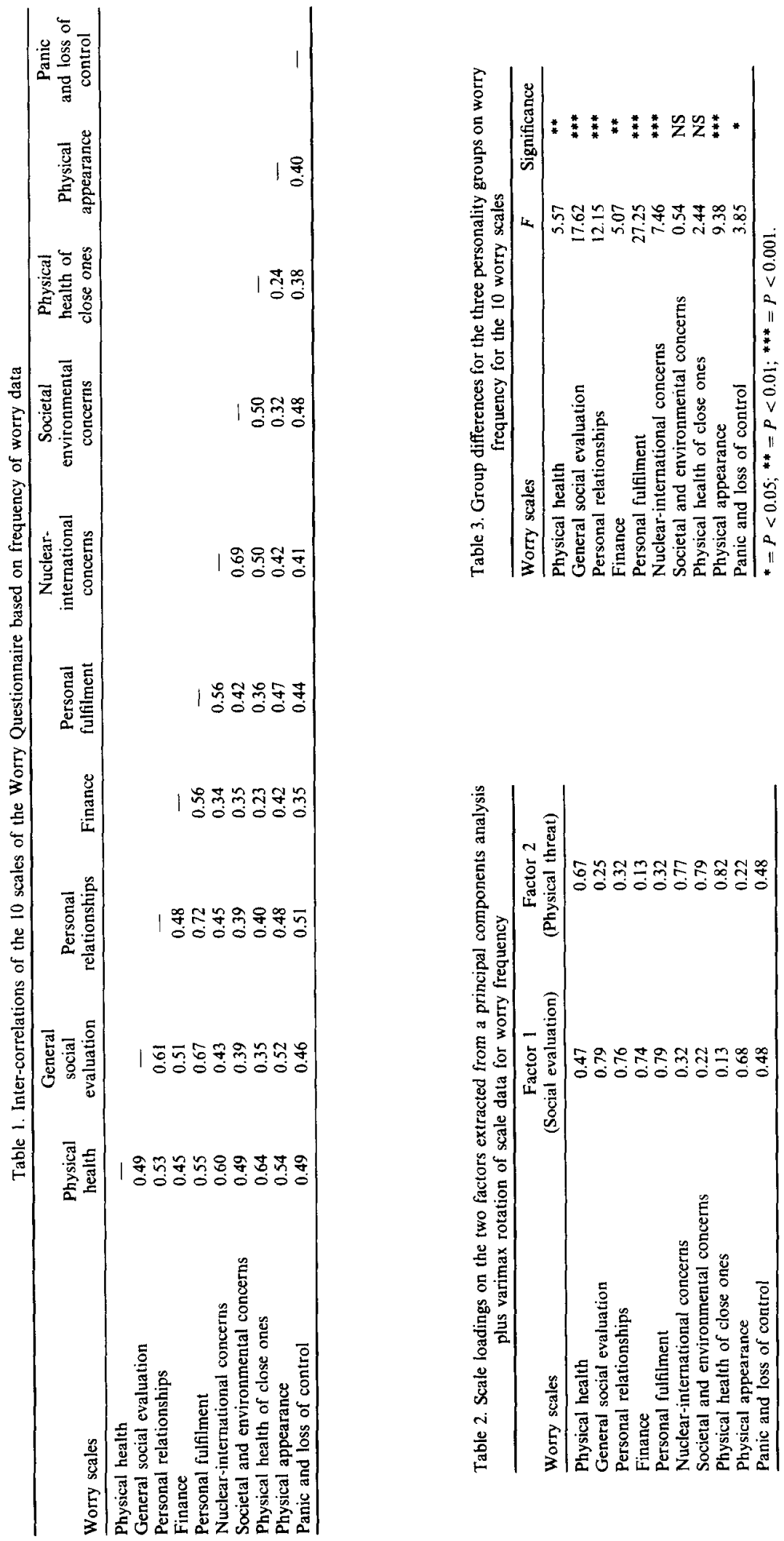


\begin{tabular}{|c|c|}
\hline Worry scales & Function 1 \\
\hline Physical health & 0.40 \\
\hline General social evaluation & 0.58 \\
\hline Personal relationships & 0.55 \\
\hline Finance & 0.46 \\
\hline Personal fulfilment & 0.81 \\
\hline Nuclear-international concerns & 0.40 \\
\hline Societal and environmental concerns & 0.12 \\
\hline Physical health of close ones & 0.17 \\
\hline Physical appearance & 0.58 \\
\hline Panic and loss of control & 0.33 \\
\hline
\end{tabular}

group was also non-significant, $F(2,59)=0.99$. In contrast, there was a highly significant effect of personality group, $F(2,59)=14.56, P<0.001$. Further analysis of this main effect indicated that the low-anxious and repressor groups did not differ significantly in mean worry frequency (means of 1.19 and 0.98 , respectively), but that the high-anxious group had a significantly higher mean worry frequency (2.23) than either of the other two groups.

The group differences in worry frequency for each of the ten worry scales or domains were then considered. As can be seen in Table 3, there were significant group differences on every worry scale except for physical health of close ones and societal and environmental concerns. A discriminant analysis was then carried out on the frequency of worry scores for each scale for the three personality groups. Variables were included in stepwise fashion minimizing Wilks lambda. This analysis yielded one significant discriminant function, $\chi^{2}=48.3, d f=6, P<0.001$. The correlations between this discriminant function and worry scale scores are shown in Table 4 . Those scales which discriminate best among the three personality groups are general social evaluation, personal relationships, personal fulfilment, finance, and physical appearance. It is noteworthy that these five scales are the scales which load most heavily on the major social evaluative factor (see Table 2). To decide which groups differ in this fashion, the group centroids on the first discriminant function were computed. The average scores on this function for each group were as follows: -0.39 for the low-anxious group; -1.09 for the repressor group; and 1.52 for the high-anxious group. These scores indicate that the first discriminant function primarily discriminates between the high-anxious and the other two groups.

Two more multivariate analyses were conducted on the low-anxious and repressor groups only. The multivariate group main effect was not statistically significant, $F(10,31)=1.30$. In addition, the one emerging discriminant function was also not significant, $\chi^{2}=11.7, d f=4$. The implication of these findings is that the reported worry frequencies of the low-anxious and repressor groups are highly similar.

\section{DISCUSSION}

The findings from this study are relevant to the issue of the number and nature of worry domains. The principal components analysis of the ten worry scales produced only two major factors or worry domains: social evaluation and physical threat. In similar fashion, the first two factors that emerged from a principal components analysis of all of the items in the questionnaire related to the social evaluation and physical threat domains. However, in this latter analysis, there was a small third factor relating to societal problems. It is noteworthy that five out of the six worry domains identified by Tallis $e t$ al. (1992) clearly relate to social evaluation, which confirms the importance of social evaluation as the single most important worry domain.

There were clear-cut effects of trait anxiety on worry. Overall, there was a highly significant correlation of +0.65 between trait anxiety and worry frequency, which replicates the earlier findings of Borkovec et al. (1983) and Tallis et al. (1992). While defensiveness as measured by the Marlowe-Crowne Social Desirability Scale was negatively correlated with worry frequency, there were no differences in worry between the low-anxious and repressor groups. However, as had been anticipated, repressors reported slightly lower worry frequency then low-anxious individuals on most of the scales. It is possible that repressors under-reported their actual worry frequencies, but this issue cannot be addressed by the data collected in this study. 
When the three personality groups were compared for frequency of worry on each of the ten worry scales, significant group differences were obtained with respect to eight of the scales. This suggests that those high in trait anxiety characteristically worry more about most concerns than do those low in trait anxiety. However, in spite of the fact that the high-anxious group generally reported much higher worry frequency than the other two groups, the three groups did not differ in worry frequency on the physical health of close ones and the societal and environmental concerns scales. A more precise account of the effects of trait anxiety on worry frequency is available as a result of the identification of the two major underlying factors of social evaluation and physical threat. The differences between those high and low in trait anxiety were considerably greater for social evaluation worries than for physical threat worries, presumably because anxiety about social evaluation is of fundamental importance to trait anxiety

There is some confirmatory evidence in the literature for the above contention. Endler, Magnusson, Ekehammar and Okada (1976) carried out a factor analysis of Spielberger's State-Trait Anxiety Inventory, the Behavioural Reactions Questionnaire, and the S-R Inventory of Anxiousness. This produced two major factors which they labeled inter-personal threat and physical danger. Trait anxiety loaded approx. 0.80 on the inter-personal threat factor, but it loaded only 0.25 on the physical danger factor.

The notion that the cognitive concerns of anxious individuals relate mainly to social and physical threats has received some support from studies of clinically anxious patients. Beck, Laude and Bohnert (1974) obtained reports from generalized anxiety disorder patients at times of increased anxiety. The reported thought content invariably involved personal danger. Sometimes the danger was predominantly physical in nature (e.g. assault), and sometimes it was social in nature (e.g. failure). Hibbert (1984) carried out a similar study, but he made use of a rather more standardized form of questioning. He replicated the finding of Beck et al. (1974) that the ideation of clinically anxious patients at particularly anxious times typically concerns social or physical threats.

Acknowledgements--Many thanks are due to Professor Andrew Mathews and Dr Colin MacLeod for the unpublished worry questionnaire which served as the starting point for the Worry Questionnaire used in this study.

\section{REFERENCES}

Barlow, D. H. (1988). Anxiety and its disorders. New York: Guildford Press

Beck, A. T., Laude, R. \& Bohnert, M. (1974). Ideational components of anxiety neurosis. Archives of General Psychiatry, $31,319-325$

Borkovec, T. D., Robinson, E., Pruzinsky, T. \& DePree, J. A. (1983). Preliminary exploration of worry: Some characteristics and processes. Behaviour Research and Therapy, 21, 9-16.

Craske M. G., Rapee, R. M., Jackel, L. \& Barlow, D. H. (1989). Qualitative dimensions of worry in DSM-111-R generalized anxiety disorder subjects and nonanxious controls. Behaviour Research and Therapy, 27, 397-402.

Crowne, D. \& Marlowe, D. (1964). The approval motive. New York: Wiley,

Endler, N. S., Magnusson, D., Ekehammar, B. \& Okada, M. (1976). The multidimensionality of state and trait anxiety. Scandinavian Journal of Psychology, 17, 81-96.

Eysenck, M. W. (1992). Anxiety: The cognitive perspective. London: Lawrence Erlbaum.

Eysenck, M. W. \& Mathews, A. (1987). Trait anxiety and cognition. In Eysenck, H. J. \& Martin, I. (Eds), Theoretical foundations of behaviour therapy. New York: Plenum.

Hibbert, G. A. (1984). Ideational components of anxiety: Their origin and content. British Journal of Psychiatry, 144, 618-624.

Meyer, T. J., Miller, M. L., Metzger, R. L. \& Borkovec, T. D. (1990). Development and validation of the Penn State Worry Questionnaire. Behaviour Research and Therapy, 28, 487-495.

Schwartz, G. E. (1983). Disregulation theory and disease: Applications to the repression/cerebral disconnection/ cardiovascular disorder hypothesis. International Review of Applied Psychology, 32, 95-118.

Spielberger, C. D. (1983). Manual for the State-Trait Anxiety Inventory (Form Y). Palo Alto, CA: Consulting Psychologists Press.

Steptoe, A. \& Kearnsley, N. (1990). Cognitive and somatic anxiety. Behaviour Research and Therapy, 28, 75-81.

Tallis, F., Eysenck, M. W. \& Mathews, A. (1992). A questionnaire for the measurement of nonpathological worry. Personality and Individual Differences, 13, 161-168.

Weinberger, D. A., Schwartz, G. E. \& Davidson, J. R. (1979). Low-anxious, high-anxious, and repressive coping styles: Psychometric patterns and behavioural and physiological responses to stress. Journal of Abnormal Psychology, 88, 369-380. 